\title{
Photoionization dynamics in the presence of attosecond pulse trains and strong fields
}

\author{
Niranjan Shivaram ${ }^{\mathrm{a}}$, Henry Timmers ${ }^{\mathrm{a}}$, Xiao-Min Tong ${ }^{\mathrm{b}}$, Arvinder Sandhu $^{\mathrm{a}}$ \\ ${ }^{a}$ Department of Physics and College of Optical Sciences, University of Arizona, Tucson, AZ, 85721 USA. \\ ${ }^{b}$ Center for Computational Sciences, University of Tsukuba, Ibaraki 305-8573, Japan.
}

\begin{abstract}
We present experimental results and a theoretical framework for understanding the ionization dynamics in atoms exposed to XUV attosecond pulse trains and strong multi-cycle infrared (IR) fields. We invoke the Floquet formalism to model dressed atomic states as a manifold of Fourier components spaced by the laser frequency. In XUV-IR pump-probe measurements, we observe that the ionization yield oscillates due to quantum interference between photo-excitation paths to a Floquet state. We show that the intensity-dependent shifts of atomic structure modify the ionization channels and the associated interference phase. We extract this phase variation and compare it with simulations. These results provide a comprehensive description of the two-color ionization process and enable new schemes for control of attosecond ionization and fragmentation dynamics.
\end{abstract}

Keywords: Attosecond, Strong fields, Photoionization, Floquet

\section{Introduction}

The roots of attosecond science [1] lie in the strong-field phenomena of high-harmonic generation (HHG) [2]. Actually, attosecond science is tied to strong-field physics in more than one way. For example, attosecond spectroscopy often requires synchronized strong $\left(10^{11}-10^{13} \mathrm{Wcm}^{-2}\right)$ laser fields as a probe of electron dynamics. This requirement arises due to the fact that low flux attosecond XUV pulses prepare a very weakly excited sample, and to obtain useful statistics the probe needs to have a very high photon flux. Thus, both attosecond XUV sources and femtosecond strong-field sources are intricately connected in the attosecond spectroscopy scheme. This unique combination has resulted in new insights, both in the study of highlyexcited atomic and molecular systems $[3,4,5]$ and the transient non-perturbative dynamics such as tunneling ionization [6].

Here, we present a comprehensive description of interaction of atoms with XUV attosecond pulse trains and moderately intense IR pulses. Our results provide insight into the strongfield modification of atomic and molecular structure and open up new approaches for the control of electron dynamics. We begin with an attosecond-resolved case study of two-color ionization dynamics of Helium atoms to obtain quantitative information about interferences in photoionization paths. As the IR intensity changes, the ionization channels get modified. We find that the yield from each atomic resonance-mediated ionization channel oscillates with a specific phase, which can be interpreted using the Floquet interaction model. We use our approach to infer not only the ionization dynamics, but also certain hard to obtain properties of the attosecond pulse train (APT), such as the absolute timing of XUV bursts relative to the driving IR fields. Numerical time-dependent Schrödinger

Email address: sandhu@physics .arizona.edu (Arvinder Sandhu) (a)

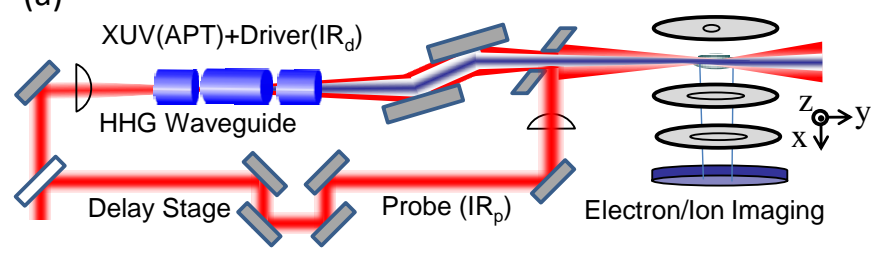

(b)

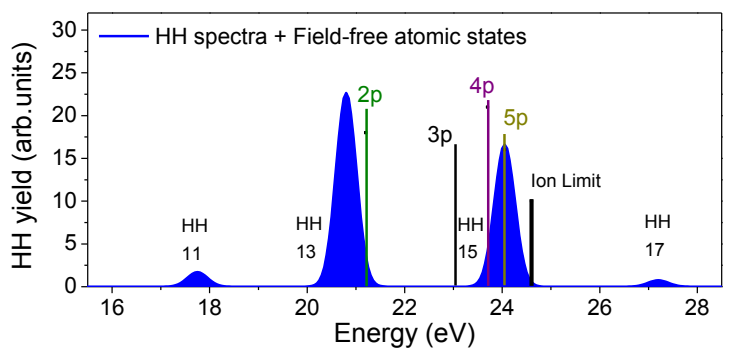

Figure 1: (a) Experimental setup showing XUV-APT beam, IR beams and the photo-fragment detection scheme. (b) XUV spectrum relative to the He atomic states in the absence of external fields.

equation (TDSE) calculations are used for comparison with experimental results and they also serve to verify the underlying mechanisms. We use this knowledge to show that strong fields of appropriate strength and timing can be utilized to control atomic and molecular phenomena.

\section{Experimental details}

In our experimental setup, we obtain amplified $65 \mathrm{fs}, 785 \mathrm{~nm}$ IR pulses of $1.5 \mathrm{~mJ}$ energy from a Ti:Sa laser amplifier. As shown in figure 1(a), the IR pulse is split into two parts. One pulse of intensity $\sim 6 \times 10^{13} \mathrm{Wcm}^{-2}$ is focused into a Xenon 
(a)

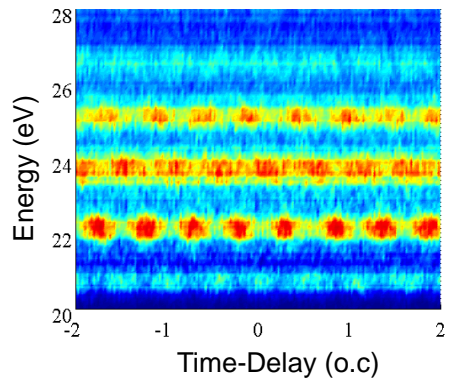

(b)

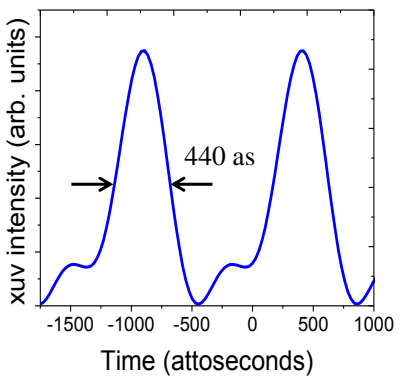

Figure 2: (a) Temporal characterization of APT using IR induced continuum interferences that result in sidebands (b) Temporal width of XUV bursts obtained from the sideband phases.

filled waveguide to drive high-harmonic generation in the form of an APT. The APT is focused onto an effusive Helium gas jet using a toroidal mirror. The second pulse $\left(\mathrm{IR}_{p}\right)$ goes to a precision delay stage and is then focused with a $50 \mathrm{~cm}$ lens onto the He target in the interaction region leading to a peak intensity $\sim 3 \times 10^{12} \mathrm{Wcm}^{-2}$. The XUV and IR fields are linearly polarized along the $\mathrm{z}$-direction and propagate along the $\mathrm{y}$-direction. The photo-fragments resulting from the interaction are accelerated along the $\mathrm{x}$-direction and imaged onto the detector by applying suitable voltages on annular metal electrodes. The detector consists of a micro-channel plate (MCP) stack backed by phosphor screen. The images from the phosphor are obtained using collection optics and a sensitive CCD camera. The photoelectrons are imaged onto the MCP in a velocity map imaging scheme (VMI) [7]. The $\mathrm{He}^{+}$ions are imaged using a spatially selective Gouy phase gating technique which allows us to eliminate averaging due to Gouy phase slip [8]. This method provides a precise measurement of ionization dynamics.

Figure 1(b) shows the high-harmonic $(\mathrm{HH})$ spectra that we utilize in our experiment relative to the unperturbed atomic states of the Helium atom. There are only two dominant harmonics $\left(13^{\text {th }}\right.$ and $\left.15^{\text {th }}\right)$ in the XUV spectrum. In the field-free configuration, $15^{\text {th }}$ harmonic is resonant with the $5 p$ electronic state and $13^{\text {th }}$ harmonic is slightly below the $2 p$ resonance. The other two harmonics that we observe, i.e. $11^{\text {th }}$ and $17^{\text {th }}$, are much weaker and non-resonant, hence they do not play a significant role in our study.

We characterize the temporal profile of our attosecond pulses using the RABBITT technique $[9,10]$. The XUV APT generated in Xe photoionizes Ar and a weak probe IR leads to onephoton interferences that show up as sidebands in the RABBITT scan (Fig.2(a)). Figure 2(b) shows the attosecond bursts reconstructed from the knowledge of phases of various sidebands. The XUV bursts in our case are approximately 440 attoseconds, which is expected for a pulse train constructed from just two main harmonics.

\section{Results and Discussion}

We apply the APT and moderately strong IR pulse to study attosecond resolved ionization dynamics of Helium. The (a)

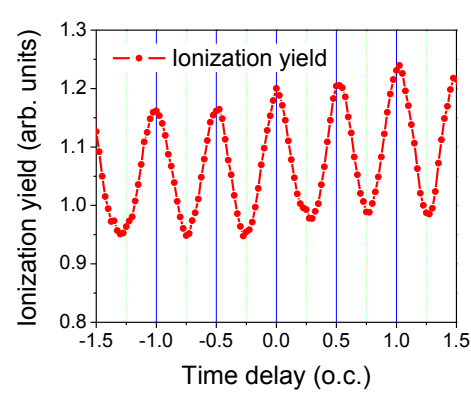

$2 \omega$ oscillation with XUV-IR time delay

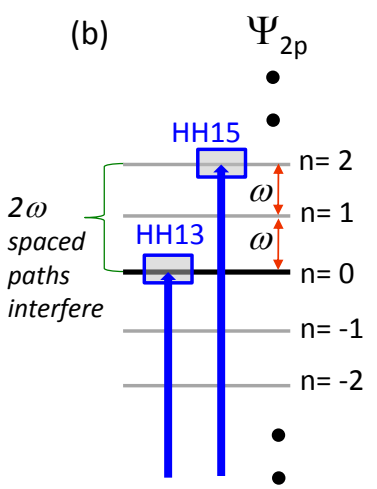

Figure 3: (a) The He ionization yield measured using $\mathrm{APT}$ and $\mathrm{IR}_{p}$ pulses as a function of time delay between them. The intensity of $\mathrm{IR}_{p}$ was $\sim 2 \mathrm{TWcm}^{-2}$. (b) Laser dressed Floquet manifold corresponding to the $2 \mathrm{p}$ state and the two harmonics used in our experiment. The interference between two Fourier paths separated by $2 \omega$ explains the observed half-cycle oscillations in the ionization yield.

physics of two-color XUV+IR ionization dynamics is very rich and multiple studies have recently explored different facets of this problem $[11,12,13,14]$. For example, the phase of twophoton transition relative to a direct continuum transition has been measured [11] as the detuning between the harmonic photon energy and the intermediate resonant state is varied. The XUV transparency in an IR laser modified atom has been discussed [12]. In this paper, we investigate the dynamically changing ionization channels and the quantum mechanical origin of the phases associated with these channels. We obtain quantitative measurements of the interference phase manifested in the ion-yield oscillations and investigate its variation in terms of the evolution of the laser-dressed atomic structure.

\subsection{Interfering paths in the Floquet picture}

We begin by describing interferences that occur in two-color photoionization. Time-resolved pump-probe ionization measurements, using APT consisting of sub-threshold harmonics and strong IR fields, by us (Fig.3(a)) and others [15, 16] show that $\mathrm{He}^{+}$ion yield oscillates as a function of time delay with half-IR-cycle periodicity. This has been interpreted as interference between wave packets generated by successive bursts in the APT $[15,17,13]$. Here, we explain the mechanisms underlying this interference process using a Floquet interaction picture [18].

The dynamics of an atom in a periodic external field are governed by the following time-dependent Schrödinger equation (in atomic units):

$$
i \frac{\partial \psi(t)}{\partial t}=\left[H_{0}+V(t)\right] \psi(t)
$$

$H_{0}$ is the field free atomic potential and $V(t)$ is a periodic external potential. In the Floquet formalism [18], the solution can be written in the form

$$
\psi_{\alpha}(t)=e^{-i \epsilon_{\alpha} t} \sum_{n=-\infty}^{n=+\infty} e^{-i n \omega t} \phi_{\alpha, n}
$$


where $\epsilon_{\alpha}$ is the complex quasi-energy, and its imaginary part is $-\Gamma / 2$ with $\Gamma$ the width (or ionization rate) of the Floquet states, $\phi_{\alpha, n}$ is the time-independent wavefunction for the nth Fourier component of the $\alpha$ Floquet state and $\omega$ is the frequency of the external periodic field. Thus, in the presence of a laser field, electronic structure can be conveniently described by Floquet states, where each bound state is associated with many onephoton $(\omega)$ spaced sidebands or Fourier components. As an example, the Floquet manifold corresponding to the $2 \mathrm{p}$ atomic state is shown in figure 3(b).

If the XUV APT arrives at a time delay $\tau$ relative to the IR field, we can write the XUV field as

$$
E_{x}=\int f\left(\omega_{x}\right) e^{-i \omega_{x}(t+\tau)} d \omega_{x}
$$

where $f\left(\omega_{x}\right)$ represents the energy content of the XUV pulse (i.e. the high-harmonic spectral amplitude). If the external field is strong enough to modify the excited and continuum states, but weak enough not to perturb the ground state, the transition amplitude from the ground state to a Floquet state in the IR field can be written as

$$
M_{g \rightarrow \alpha}=\int\left\langle\psi_{\alpha}(t)\left|z E_{x}\right| \psi_{g}(t)\right\rangle d t
$$

Substituting for $\psi_{\alpha}(t)$ from equation 1 and $E_{x}$ from equation 2 and implementing integration, we obtain the transition probability as

$$
P_{\alpha}(\tau)=\left|\sum_{n}\left\langle\phi_{\alpha n}|z| \phi_{g}\right\rangle f\left(\omega_{n}\right) e^{-i \omega_{n} \tau}\right|^{2}
$$

where $f\left(\omega_{n}\right)$ is the XUV field strength at the energy corresponding to the $\mathrm{n}^{\text {th }}$ Fourier component of the excited Floquet state, namely at energy $\omega_{n}=\operatorname{Re}\left(\epsilon_{\alpha}\right)+n \omega-\epsilon_{g}$.

In the case of only two harmonics causing atomic excitation, we have only two non-zero matrix elements. For example, the $13^{\text {th }}$ and $15^{\text {th }}$ harmonics used in our experiment cause transitions to $n=0$ and $n=2$ components of the $2 p$ Floquet manifold shown in Fig. 3(b). In such a case, the transition probability of equation 4 can be simply expressed as

$$
P(\tau)=\left|M_{0} f_{0}+M_{2} f_{2} e^{-i(2 \omega \tau+\phi)}\right|^{2}
$$

where $M_{0}$ and $M_{2}$ are the two transition matrix elements and $f_{0}$ and $f_{2}$ are the strengths of the two harmonics at the energies corresponding to the $n=0$ and $n=2$ components of a given Floquet state. The matrix elements are real and can either be positive or negative. The relative sign of matrix elements is included in this expression through $\phi$, which can take values 0 or $\pi$.

Equation 5 represents two excitation paths and the interference between these two paths modulates the excitation probability with time delay at $2 \omega$ frequency $[19,20]$. The ionization probability, which is proportional to the excitation probability, thus also exhibits an oscillatory variation at $2 \omega$ or half-cycle periodicity with time-delay. This explains the origin of ion and electron yield oscillations and also provides an avenue to obtain quantitative information about the phases of different ionization channels.
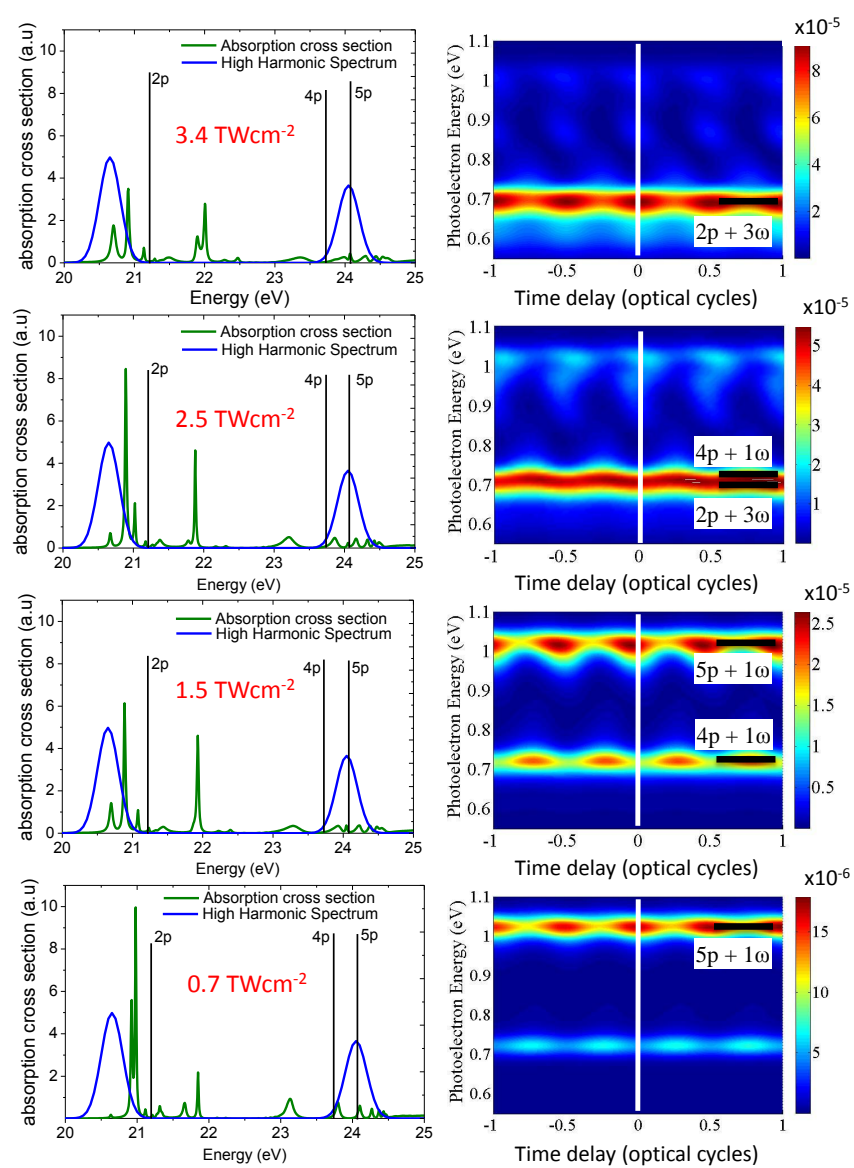

Figure 4: (a) Calculated XUV photoabsorption cross-section in atomic units as a function of photon energy at four IR intensities and the high-harmonic spectrum showing the $13^{\text {th }}$ and $15^{\text {th }}$ harmonics. Field-free atomic states are shown as vertical lines. (b) TDSE simulation results showing time-resolved photoelectron spectra for the high harmonic spectrum used in our experiment at the four probe IR intensities. The white line marks time-delay zero and helps in identifying the phase of the electron yield oscillations. The labels on the right identify the origin of photoelectrons in terms of the intermediate atomic state.

\subsection{Intensity dependent ionization channels: Simulation}

The two-color $(X U V+I R)$ ionization channels are dynamically dependent on the strength of the IR field acting on the atom. This intensity dependence of ionization channels can be understood from figure 4, which plots photoabsorption cross sections of Helium atom as a function of XUV photon energy at four laser intensities (figure $4(\mathrm{a})$ ). The $13^{\text {th }}$ and $15^{\text {th }}$ harmonics are also shown. The photoabsorption cross section plots were calculated using the method described in [21]. Figure 4 (b) shows XUV-IR $p$ time delay dependent photoelectron energy distributions obtained by TDSE simulations for the same four intensities and harmonic spectrum.

In figure 4(a), the green line shows the structures that develop in the absorption cross section due to the presence of the IR field. Even for a moderately strong IR field, atomic states get shifted and broadened and new structures develop. For example, as the intensity is increased, the $2 p$ state which is located at $21.2 \mathrm{eV}$ in the unperturbed atom, gets shifted towards lower energies and develops into multiple peaks. Similarly, the $4 p$ and 


\begin{tabular}{c|c} 
Floquet state & Phase \\
\hline \hline $2 \mathrm{p}$ & $0,2 \pi$ \\
$4 \mathrm{p}$ & $\pi$ \\
$5 \mathrm{p}$ & $0,2 \pi$
\end{tabular}

Table 1: The phase difference $\phi$ between transition matrix elements to Fourier components of Floquet states for the three cases relevant to our measurements.

$5 \mathrm{p}$ states get ponderomotively shifted to higher energies and get broadened as the intensity is increased. Thus, from figure 4(a) it is apparent that even under moderately intense laser fields $\left(\sim 10^{12} \mathrm{Wcm}^{-2}\right)$, the discrete atomic resonances evolve into a complicated structure.

As a result of the intensity dependent modification of atomic structure, with change of IR laser intensity, different ionization channels dominate the ionization process at different intensities. At the highest intensity, the $2 \mathrm{p}$ structure is resonant with the $13^{\text {th }}$ harmonic and dominates photoabsorption. However, at the lowest intensity, the $2 \mathrm{p}$ structure is non-resonant. In this case, the $4 p$ and $5 p$ structures which are resonant with the $15^{\text {th }}$ harmonic can dominate the photoabsorption.

Figure 4(b) shows that the photoelectron yields oscillate with XUV-IR $p$ time delay at the expected $2 \omega$ frequency. At each intensity, many Floquet ionization channels are active and they exhibit different oscillation phase. At the highest intensity, the Floquet channel mediated by the $2 \mathrm{p}$ resonance producing photoelectrons with $0.69 \mathrm{eV}$ energy is dominant. In terms of the multiphoton picture, this represents three-photon ionization of the XUV excited $2 \mathrm{p}$ state. Thus, we label this as the $2 p+3 \omega$ channel. At the lowest intensity, we note that the $5 p+1 \omega$ channel becomes dominant and the $2 p+3 \omega$ channel is almost closed. The $4 p+1 \omega$ channel also provides substantial contribution to ionization at lower intensities. This switching between dominant ionization channels agrees with the laser induced modification of atomic structure in figure 4(a).

Importantly, the delay-dependent $2 \omega$ oscillations in figure 4(b) show that the $2 p+3 \omega$ channel and the $5 p+1 \omega$ channel exhibit a peak ionization at zero time delay, whereas the $4 p+1 \omega$ channel exhibits a minimum at zero time delay. This indicates that the quantum phase difference between Fourier components, represented by $\phi$ in equation 5 is different for different ionization channels. The value of $\phi$ for both the $2 p+3 \omega$ channel and the $5 p+1 \omega$ channel is 0 (or $2 \pi$ ), whereas for the $4 p+1 \omega$ channel (at $0.72 \mathrm{eV}$ ) it is $\pi$. The phases associated with different ionization channels are summarized in table 1.

As mentioned above, the values of $\phi$ for the $4 p$ and $5 p$ Floquet channels are $\pi$ and 0 respectively. This difference is intriguing since the unperturbed $4 \mathrm{p}$ and $5 \mathrm{p}$ states are close lying Rydberg states with similar properties. In order to gain more insight into this difference in the values of $\phi$ for the $4 p$ and $5 p$ states, we perform theoretical calculations of $\phi$ in XUV + IR photo-ionization at two IR wavelengths. In this calculation, the XUV field is given by

$$
E_{x}^{ \pm}=\cos \left(\omega_{x} t\right) \pm \cos \left(\left(\omega_{x}-2 \omega\right) t\right)
$$

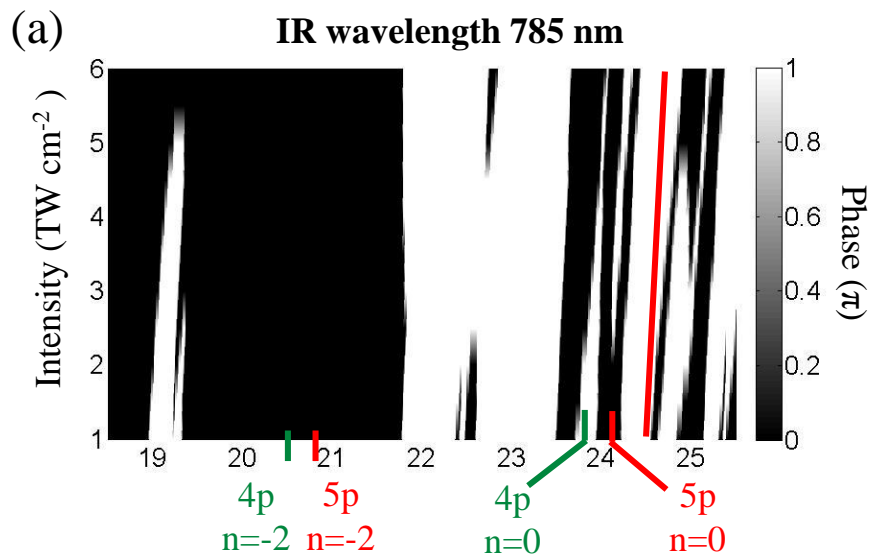

Energy of the $\omega_{\mathrm{x}}$ photon $(\mathrm{eV})$

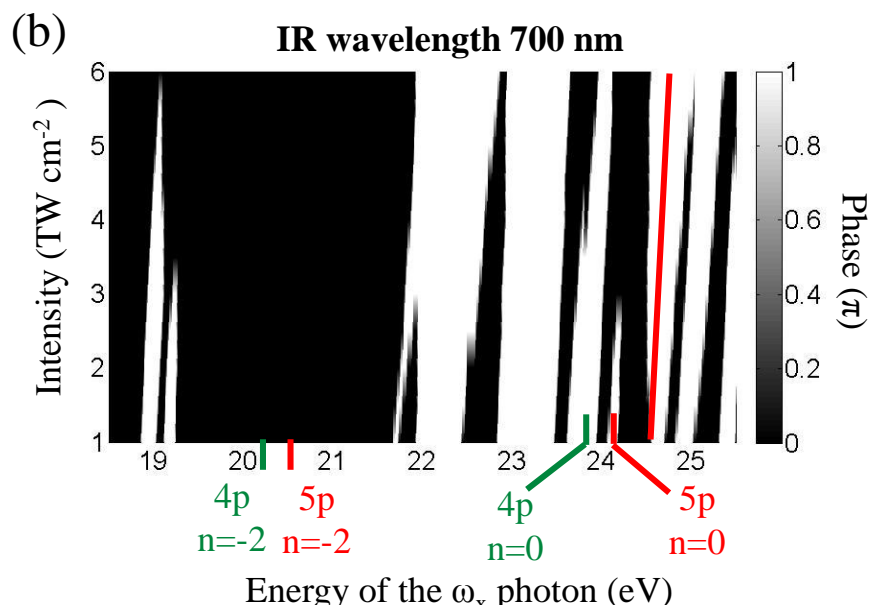

Figure 5: (a) Phase difference between matrix elements of transitions induced by two harmonics of frequency $\omega_{x}$ and $\omega_{x}-2 \omega$ as a function of $\omega_{x}$ and IR intensity for an IR wavelength of $785 \mathrm{~nm}$. (b) The phase difference plot for an IR wavelength of $700 \mathrm{~nm}$. The $n=0$ and $n=-2$ Fourier components of the $4 \mathrm{p}$ and 5p Floquet states are also shown. The slanted red line starting from 24.6 $\mathrm{eV}$ represents the ionization threshold.

where $\omega$ is the frequency of the IR field. For this case, from equation (5), the probability of ionization is given by

$$
P(\tau)^{ \pm}=\left|M_{x} \pm M_{x-2} e^{-i(2 \omega \tau+\phi)}\right|^{2}
$$

$M_{x}$ and $M_{x-2}$ are the matrix elements of transitions induced by the $\omega_{x}$ and $\omega_{x}-2 \omega$ photons respectively. Also, $P(\tau)^{+}$is the ionization probability corresponding to $E_{x}^{+}$and $P(\tau)^{-}$is the ionization probability corresponding to $E_{x}^{-}$.

To obtain $\phi$ as a function of $\omega_{x}$ and IR laser intensity, we define

$$
S\left(\omega_{x}\right)=\frac{P^{+}-P^{-}}{\left|P^{+}-P^{-}\right|}
$$

which takes values +1 or -1 . $P^{+}$and $P^{-}$are the ionization probabilities at zero time-delay. Since the phase $\phi$ is 0 or $\pi$, we can extract $\phi$ by plotting $(1-S) \pi / 2$ as a function of $\omega_{x}$ and IR intensity.

As mentioned earlier, the unperturbed $4 p$ and $5 p$ state wavefunctions are expected to have similar properties. At low intensities, the $n=0$ Fourier components of the $4 \mathrm{p}$ and $5 \mathrm{p}$ Floquet 
states also behave similarly. Hence, we make a hypothesis that the difference in values of $\phi$ for the $4 p$ and $5 p$ Floquet states may arise from a difference in the properties of the wavefunctions of their $n=-2$ components. To test this hypothesis, we plot the phase $\phi$ obtained using the procedure described above, at two different IR wavelengths - $785 \mathrm{~nm}$ and $700 \mathrm{~nm}$. Changing the wavelength of the IR field allows us to change the energy location of the $n=-2$ Fourier component while keeping the $n=0$ component fixed, for a given Floquet state. Figure 5 shows a plot of phase $\phi$ as a function of $\omega_{x}$ and IR intensity for the two IR wavelengths. It should be noted that in this figure, the phase at a given $\omega_{x}$ is the phase difference between excitation matrix elements $\left(M_{x}\right.$ and $\left.M_{x-2}\right)$ for photon energies of $\omega_{x}$ and $\omega_{x}-2 \omega$. The location of the $n=0$ and $n=-2$ Fourier components of the $4 p$ and $5 p$ Floquet states at the lowest intensity of $1 \mathrm{TWcm}^{-2}$ are also shown in figure 5 . As the intensity is increased, due to the ponderomotive effect, these Floquet states shift upwards in energy, parallel to the slanted red line which represents the ionization threshold. By comparing figures 5(a) and $5(\mathrm{~b})$ at intensities of a few $\mathrm{TWcm}^{-2}$, it can be seen that the phase $\phi$ for the $5 \mathrm{p}$ Floquet state changes from zero at an IR wavelength of $785 \mathrm{~nm}$ to $\pi$ at an IR wavelength of $700 \mathrm{~nm}$. This indicates that the location of the $n=-2$ Fourier component and hence the spatial properties of its wavefunction influence the value of the phase $\phi$ for the $5 p$ Floquet state. Similarly, a difference in the values of $\phi$ for the $4 p$ and $5 p$ Floquet states at an IR wavelength of $785 \mathrm{~nm}$ (Figs. 5 (a) and 4 (b)) could arise from a difference in the spatial properties of the wavefunctions of their $n=-2$ Fourier components which critically depends on their energy location. Further experiments and simulations are necessary to obtain a better understanding of the nature of Floquet state wavefunctions and shed more light on the origin of the phases discussed here.

We now discuss our experimental measurements which serve to identify the different ionization channels and allow us to obtain the phases associated with these channels. An IR wavelength of $785 \mathrm{~nm}$ has been used in these experiments.

\subsection{Intensity dependent ionization channels: Experiment}

Two-color ionization channels are identified by the dominant Floquet state mediating the ionization. We identify the active Floquet channels using photoelectron spectroscopy at various probe peak intensities. The observed photoelectron peaks in figure 6 are associated with IR ionization of XUV excited 5p, $4 p$ and $2 p$ atomic states. At low intensities, the ionization is mediated by the $5 p$ Floquet state, and we observe a strong peak corresponding to $5 p+1 \omega$ ionization. This is expected as the $15^{\text {th }}$ harmonic is initially resonant with the $5 \mathrm{p}$ state. As the intensity is increased, the $4 p+1 \omega$ starts contributing as it comes into resonance at higher intensity. At even higher intensities the $2 p+3 \omega$ channel dominates the ionization signal. This observation is completely consistent with the simulated absorption cross section and photoelectron plots of figure 4.

The angle-resolved photoelectron images in Fig. 6(b) show that as intensity is increased towards $3.4 \mathrm{TW} \mathrm{cm}-2$, side lobes corresponding to the ' $\mathrm{g}$-wave' structure appear in the innermost photoelectron ring corresponding to the $2 p+3 \omega$ channel. This

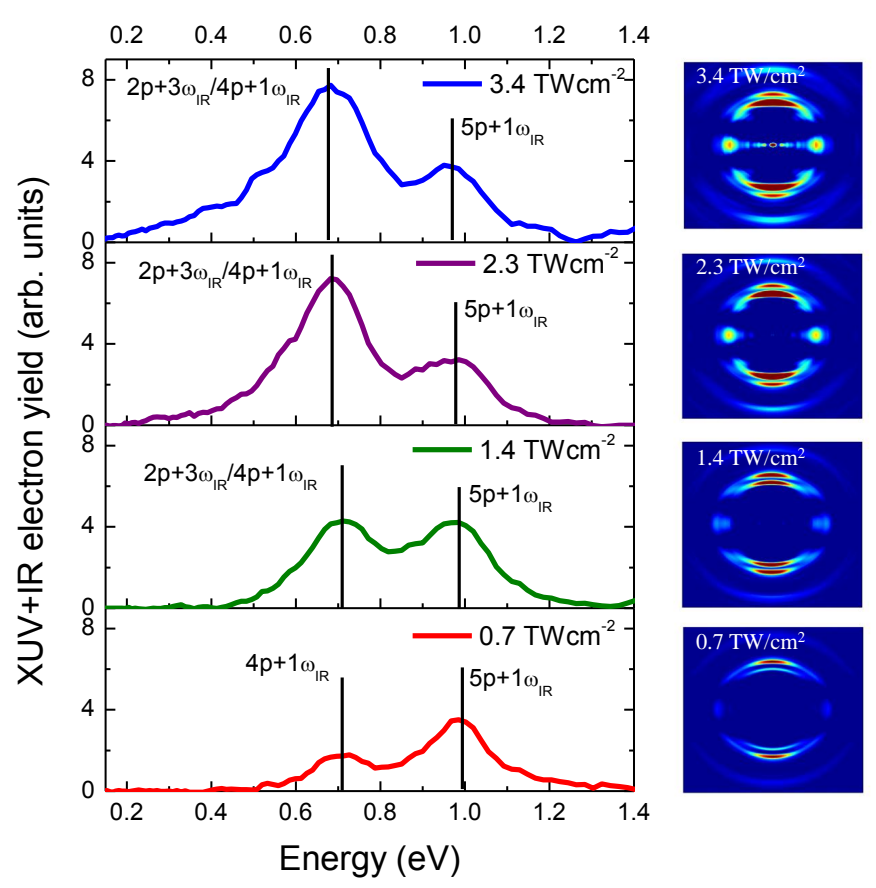

Figure 6: (a) Experimental XUV+IR $p$ photoelectron spectrum of He at different $\mathrm{IR}_{p}$ intensities. As the intensity is increased the $2 p+3 \omega$ peak becomes dominant. (b) Experimental angular distributions of photo-electrons at different $\operatorname{IR}_{p}$ intensities.

is another indication of the 3 IR-photon ionization of the XUV excited $2 p$ state. Thus, the results from Fig. 6 confirm that the dominant two-color ionization pathway changes from the $5 \mathrm{p}$ mediated ionization at low intensities to $2 \mathrm{p}$-mediated ionization signal at higher intensities.

\subsection{Phase measurement from the ion-yield oscillations}

The ionization probability corresponding to each Floquet channel is described by equation 5 with the equality sign replaced by proportionality. Hence, each ionization channel should lead to $2 \omega$ oscillations in the yield and the phase $\phi$ in the interference term of equation 5 will dictate the exact delay dependence of the ionization signal. We extract this phase term for different ionization channels from the total ion-yield and establish a quantitative relationship between the strong-field variation of atomic structure and the phase of ion-yield oscillations.

The experimental measurement of the phase of ion-yield oscillations is often complicated by interferometric drifts and experimental fluctuations. We find that a much more sensitive way to measure the phase of oscillations is by referencing it to another signal. We generate this reference signal by creating an interference between two IR pulses which results in an additional modulation in the ion-yield. One of the IR pulses is the probe pulse $\mathrm{IR}_{p}$. The second pulse, $\mathrm{IR}_{d}$ in figure 1(a), is obtained by allowing the HHG-driving IR pulse to copropagate with the harmonics exiting the waveguide. This driver $\mathrm{IR}_{d}$ propagates to the interaction chamber, where it focuses to an intensity of $\sim 10^{10} \mathrm{Wcm}^{-2}$. The driver IR pulse $\left(\mathrm{IR}_{d}\right)$ is phase locked to the APT due to the mechanism of generation. Figure 
(a)

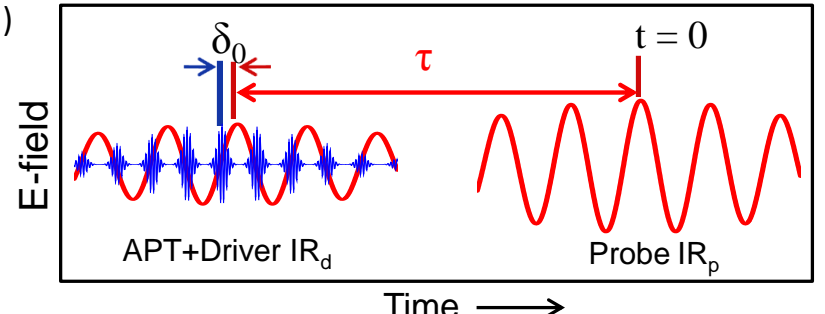

(b)

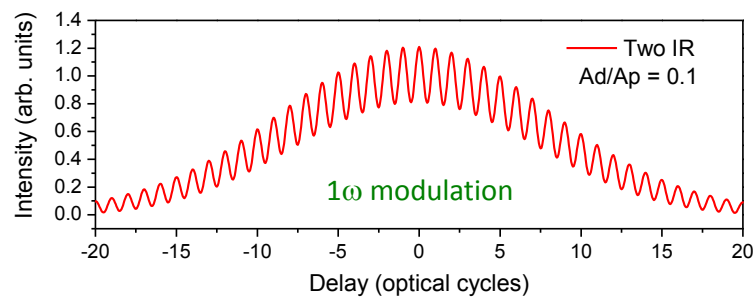

Figure 7: (a) The timing relationship between the APT, driving IR field $\left(\mathrm{IR}_{d}\right)$ and probe IR field $\left(\operatorname{IR}_{p}\right)$. The absolute timing of attosecond bursts $\delta_{0}$ is defined relative to the peak of the driving field. (b) The net IR intensity variation at the timing of XUV burst for the case of two IR pulses. The intensity modulation at $\omega$ acts as a reference in our experiment.

7 (a) defines the relative timing of the XUV and IR pulses used in our experiment and theoretical model. The interference between two IR fields leads to a net IR intensity modulation at $\omega$ frequency (Fig. 7 (b)). The phase of $2 \omega$ ionization signal can be robustly measured relative to this $1 \omega$ modulation.

Before proceeding further, it is appropriate to revisit our Floquet interaction model and include the effect of the driver IR field in the equations. For the configuration shown in Fig. 7 (a) the probe IR field, driver IR field and the net IR field respectively can be written as

$$
\begin{array}{r}
E_{p}=A_{p} \cos (\omega t) \\
E_{d}=A_{d} \cos (\omega t+\omega \tau) \\
E_{n e t}=\sqrt{I(\tau)} \cos (\omega t+\delta(\tau))
\end{array}
$$

where

$$
I(\tau)=A_{p}^{2}+A_{d}^{2}+2 A_{p} A_{d} \cos (\omega \tau)
$$

and

$$
\delta(\tau)=\arcsin \left(A_{d} \sin (\omega \tau) / \sqrt{I(\tau)}\right)
$$

The XUV field in this configuration becomes

$$
E_{x}=\int f\left(\omega_{x}\right) e^{-i \omega_{x}\left(t+\tau+\delta_{0} / \omega\right)} d \omega_{x}
$$

where $\delta_{0} / \omega$ is the time at which the attosecond bursts are locked with respect to the peak of $\mathrm{IR}_{d}$ as they come out of the gas-filled waveguide. The $\delta_{0}$ term thus provides the absolute timing of the attosecond pulses.

Proceeding as before, we can calculate the photoexcitation probability for the case of two dominant harmonics $\left(13^{\text {th }}\right.$ and $\left.15^{\text {th }}\right)$ and hence the ionization probability is obtained as

$$
P(\tau) \propto\left|M_{0} f_{0}+M_{2} f_{2} e^{-i\left(2 \omega \tau+2 \delta_{0}-2 \delta(\tau)+\phi\right)}\right|^{2}
$$

Note that the matrix elements depend on the net IR intensity $(I(\tau))$, which modulates at a frequency of $\omega$ (Fig.7(b)). Hence we can write $M_{0}^{2} \propto I(\tau)^{a}$ and $M_{2}^{2} \propto I(\tau)^{b}$ where $a$ and $b$ are real numbers. Using the approximation that $A_{d} / A_{p}<<1$ and retaining the oscillatory first order terms, equation 11 can be written as

$$
\begin{aligned}
P(\tau)=2 a \frac{A_{d}}{A_{p}} \cos (\omega \tau)+2 R \cos \left(2 \omega \tau+2 \delta_{0}+\phi\right) \\
+R \frac{A_{d}}{A_{p}}(a+b+2) \cos \left(\omega \tau+2 \delta_{0}+\phi\right) \\
\quad+R \frac{A_{d}}{A_{p}}(a+b-2) \cos \left(3 \omega \tau+2 \delta_{0}+\phi\right)
\end{aligned}
$$

where $R \approx\left(M_{2} / M_{0}\right)$ is the ratio of average or dc value of the two matrix elements. $f_{0}$ and $f_{2}$ have been ignored.

From absorption cross section calculations [21], typically, $R^{2} \approx\left(M_{2} / M_{0}\right)^{2} \leq 10^{-3}$ for the intensity range used in our experiments. Using these numbers along with $A_{d} / A_{p} \leq 0.1$, it can be seen that the first two terms in equation 12 are the dominant terms. The last two terms contain a product of $R$ and $A_{d} / A_{p}$ and are at least an order of magnitude weaker. Ignoring the last two terms, equation 12 is reduced to the following simplified expression for the probability of ionization

$$
P(\tau)=P_{1} \cos (\omega \tau)+P_{2} \cos \left(2 \omega \tau+2 \delta_{0}+\phi\right)
$$

$P_{1}$ and $P_{2}$ are the amplitudes of the two frequencies in the signal. As before, the relative sign of matrix elements is included in this expression through $\phi$, which can take values 0 or $\pi$. Next, we will obtain the value of $\phi$ for various Floquet channels active in the two-color ionization process. The term $\delta_{0}$ is also of interest as it provides the absolute timing of XUV bursts.

Figure 8(a) shows the experimental measurements of normalized $\mathrm{He}^{+}$ion-yield as a function of the time delay between $\mathrm{APT}+\mathrm{IR}_{d}$ and probe $\mathrm{IR}_{p}$ at different probe intensities. The experimental data shows distinct oscillation structure with onecycle $(1 \omega)$ and half-cycle $(2 \omega)$ variations. Figure $8(\mathrm{~b})$ plots the Fourier transform amplitudes of the experimental data shown in figure 8(a) and serves to confirm that only $1 \omega$ and $2 \omega$ frequencies are present in our ionization signal. The ionization probability given by equation 13 also implies periodic variation at $\omega$ and $2 \omega$ frequencies indicating that our model correctly captures the essence of the XUV+2IR ionization process.

The $2 \omega$ oscillation component in figure 8 is due to quantum interference between Fourier components of Floquet states. The one-cycle variation is due to the net IR intensity modulation at $\omega$ frequency. Thus, from figure 8 , we obtain sensitive measurements of the phase $\phi$ of ionization channels active at different intensities using the $1 \omega$ modulation as a reference.

Importantly, the oscillatory structure in Fig. 8 at two probe intensities, namely, $1.4 \mathrm{TW} \mathrm{cm} \mathrm{Tm}^{-2}$ and $3.4 \mathrm{TW} \mathrm{cm}^{-2}$ is very different. The asymmetric double-peak structure at $1.4 \mathrm{TW} \mathrm{cm}^{-2}$ shows the left-peak to be higher, whereas, at $3.4 \mathrm{TW} \mathrm{cm} \mathrm{cm}^{-2}$ the situation is reversed and the right-peak is higher. This difference in oscillation structure is a direct manifestation of the 
(a)

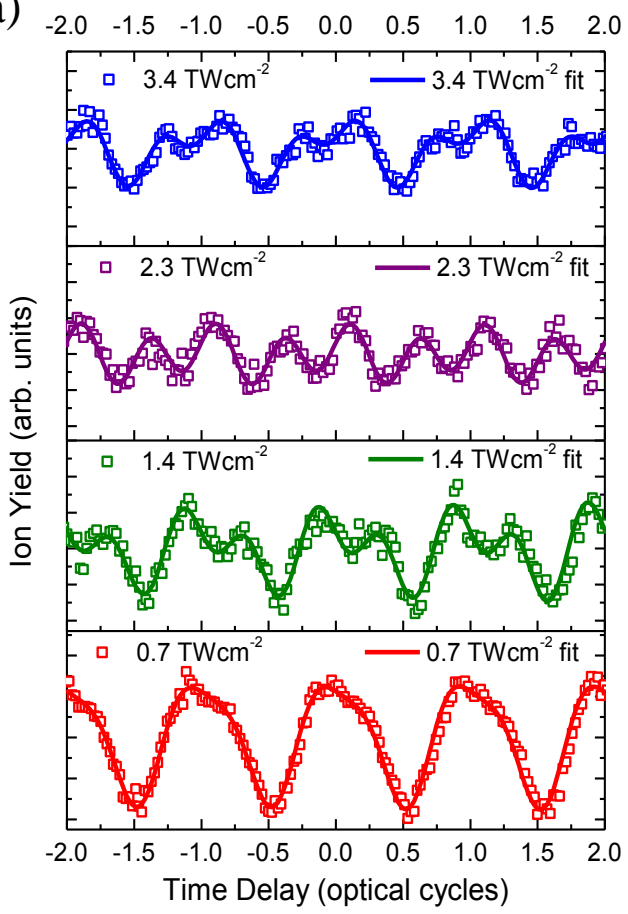

(b)

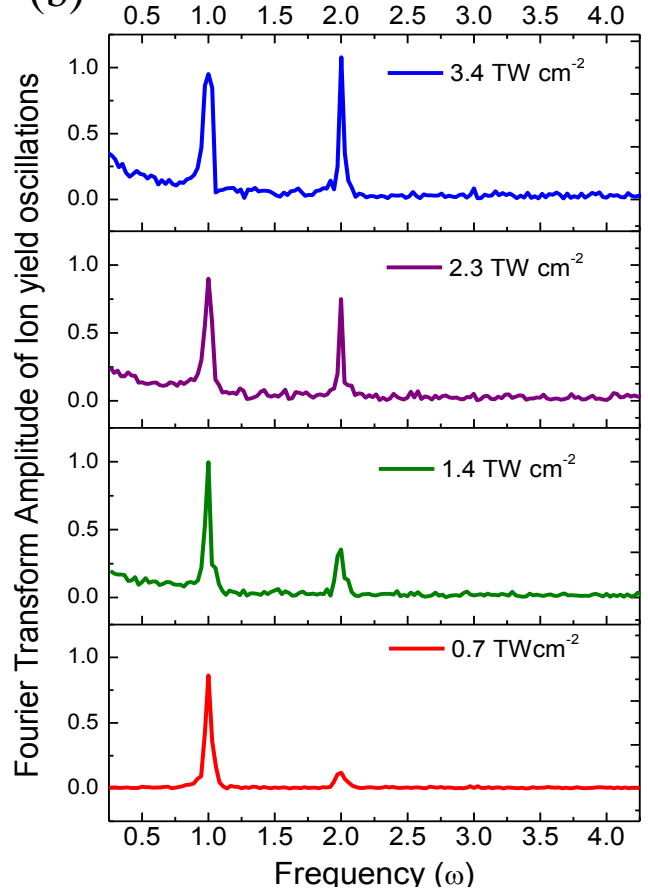

Figure 8: (a) Normalized $\mathrm{He}^{+}$ion-yield oscillations for XUV and 2IR pulses (driver and probe) at different probe peak intensities. Note that the asymmetry in the double peak oscillation structure is reversed between $1.4 \mathrm{TWcm}^{-2}$ and $3.4 \mathrm{TWcm}^{-2}$. Also shown are the fits of equation 13 to these signals. (b) The Fourier transform amplitudes of the experimental signals shown in (a). Distinct $1 \omega$ and $2 \omega$ peaks are visible in the Fourier transform. The amplitudes have been normalized to the amplitude of the $1 \omega$ peak at each intensity.

change in phase relationship between the $2 \omega$ and $1 \omega$ components. This change is attributed to the shift in the value of $\phi$ as dominant ionization channels change with intensity.

We can use equation 13 to fit the ion-yield oscillations shown in figure 8(a) and obtain the value of $\phi$ at each intensity. The solid lines in figure 8(a) show the fits for four probe IR intensities $0.7 \mathrm{TW} \mathrm{cm} \mathrm{cm}^{-2}, 1.4 \mathrm{TW} \mathrm{cm} \mathrm{cm}^{-2}, 2.3 \mathrm{TW} \mathrm{cm}^{-2}$ and $3.4 \mathrm{TW}$ $\mathrm{cm}^{-2}$. From the fits we obtain $\phi+2 \delta_{0}$ values at each intensity. The phase $2 \delta_{0}$ represents a constant offset due to the non-zero timing of attosecond bursts relative to the driver IR field (Fig. $7(a)$ ). We can use information from simulations to remove this constant offset and extract the dependence of phase $\phi$ on intensity. Referring back to figure 4, we notice that at the highest intensity of $3.4 \mathrm{TW} \mathrm{cm}^{-2}$, the $2 \mathrm{p}$ Floquet channel strongly dominates the ionization and corresponds to phase $\phi=0$ between interfering ionization paths of two harmonics (Table 1). Hence, the value of $\phi+2 \delta_{0}$ obtained from the fit to the $3.4 \mathrm{TW} \mathrm{cm}^{-2}$ data is basically just a measure of the offset $2 \delta_{0}$. The experimental fit to the $3.4 \mathrm{TW} \mathrm{cm}^{-2}$ data in figure 8 (a) thus provides $2 \delta_{0}=-2.4$ radian. We subtract this value from the $\phi+2 \delta_{0}$ values at all intensities and plot $\phi$ as a function of intensity in figure 9. The phases obtained from TDSE simulations at intensities similar to the experimental intensities are also shown. Insets in figure 9 plot the TDSE results of the ion yield oscillations in the presence of XUV and $\mathrm{IR}_{p}$ pulses as a function of time delay for two $\mathrm{IR}_{p}$ intensities $\left(3.4 \mathrm{TWcm}^{-2}\right.$ and $1.5 \mathrm{TWcm}^{-2}$ ). The experimentally extracted phases in figure 9 agree well with the phases predicted by simulations. The considerable deviation of the ex- perimental phase value at the lowest intensity of $0.7 \mathrm{TWcm}^{-2}$ from the theoretically expected value of $2 \pi$ can be attributed to errors in phase extraction arising due to the weak $2 \omega$ signal in the experimental ion yield oscillations (figure 8, red curve).

The variation of phase in figure 9 can now be related to the interference term for different ionization channels. At high intensity $\left(3.4 \mathrm{TW} \mathrm{cm} \mathrm{cm}^{-2}\right)$, the $2 \mathrm{p}$ resonance mediated ionization channel is dominant and the oscillation of this channel has zero phase. In this case the quantum phase $\phi$ between the $M_{0}$ and $M_{2}$ transition matrix elements in equation 5 (or 11) is zero. In other words, $13^{\text {th }}$ and $15^{\text {th }}$ harmonic induced transitions to the Floquet components associated with $2 \mathrm{p}$ excitation are 'in-phase' (Fig. 3(b)). As intensity decreases, the $4 p$ channel starts contributing substantially. The oscillations in this channel are out of phase with the $2 p$ contribution. In terms of the Floquet transitions, the $13^{\text {th }}$ and $15^{\text {th }}$ harmonic transition matrix elements to the components of $4 p$ Floquet state have opposite signs. At very low intensity, the $5 p$ ionization starts dominating and oscillates with the same phase as $2 \mathrm{p}$ (i.e. 0 or $2 \pi$ ). In our experiment (Fig. 9 ), as the intensity decreases, we observe a change in phase of ion-yield oscillation, going from zero when $2 p$ is dominant, through $\pi$ where $4 \mathrm{p}$ contributes substantially, and eventually towards $2 \pi$ where $5 \mathrm{p}$ dominates.

\subsection{Absolute timing of atto-bursts}

The above analysis provides a crucial piece of information that our XUV bursts are locked at a phase of $\delta_{0}=-1.2$ radian. 


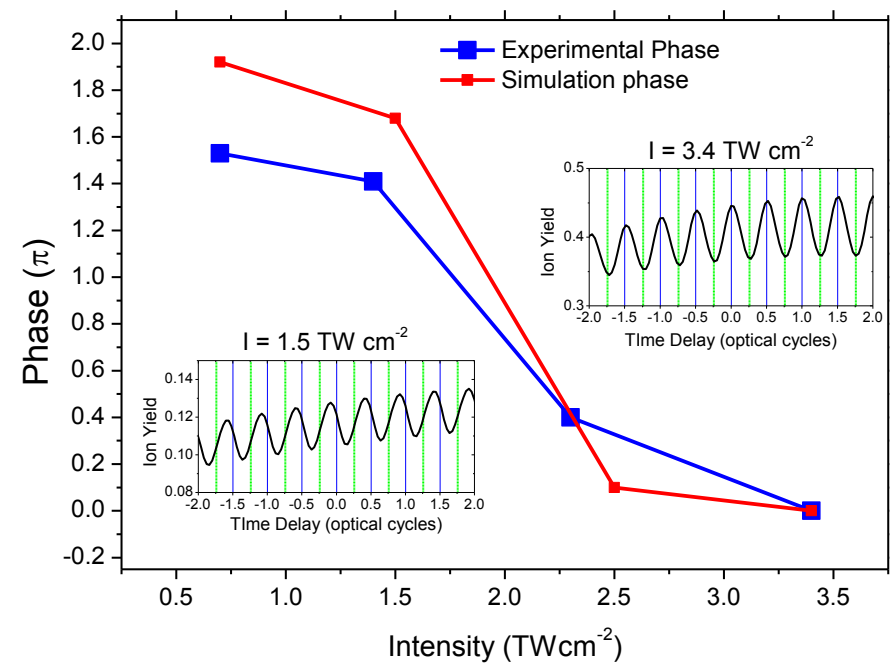

Figure 9: The experimental values of the phase $\phi$ obtained from the fits of equation 13 to the data in figure 8(a) at four different IR intensities. The phases obtained from TDSE simulations at similar intensities are also shown. The phase is 0 for the highest intensity where $2 p$ Floquet state is dominant and changes towards $2 \pi$ at lower intensities where $5 \mathrm{p}$ becomes dominant. Insets show the TDSE simulation of the net ionization yield in the presence of XUV and $\mathrm{IR}_{p}$ fields as a function of time delay at two different $\mathrm{IR}_{p}$ intensities $\left(3.4 \mathrm{TWcm}^{-2}\right.$ and $1.5 \mathrm{TWcm}^{-2}$ ).

This translates to an absolute time difference of 500 attoseconds from the nearest positive peak of the driver IR field. It is important to note that this absolute timing information is often missing in XUV+IR experiments. To extract the absolute timing, complex energy-resolved electron measurements are often required $[22,23]$. Using 2 IR pulses in two-color ionization, we have obtained the absolute timing of attosecond bursts directly from the ion-yield measurement in XUV+IR photoionization of Helium. A major simplification in our method is that it is purely based on ion-counting and does not require complicated energy-resolved measurements of electrons.

Our conclusions on the absolute timing of XUV bursts can be verified by a simple calculation of classical trajectories for near-cutoff harmonic emission in Xenon [2]. We find that the $15^{\text {th }}$ harmonic short trajectory return is at 250 degree phase relative to the negative peak of the IR field. In our measurement, the recollision time corresponding to 250 degree phase agrees well with attosecond burst located 500 attoseconds from the positive peak of the driver IR field. Furthermore, our measurement of the XUV burst timing relative to the IR field is also in qualitative agreement with the experimental results reported in reference [23].

\section{Summary}

Our theoretical and experimental work represents a detailed measurement of XUV+IR ionization dynamics. Floquet theory provides a crucial insight into these complex dynamics and explains the mechanisms underlying the quantum interferences in ionization. The knowledge of switching between ionization channels and the accompanying quantum phases opens new av- enues for control of photoionization in the presence of strong fields. A strong field with appropriately tuned intensity and timing can be used to control the interfering matrix elements and hence yield and spectral content of the photoelectrons emitted from an atom. A recent paper [12] on XUV transparency in strong fields is also based on some of these ideas. One can envision extending these concepts to study of molecular excited states which present a richer and more complex system.

Our work also highlights the fact that even a moderately strong IR field can substantially alter the ionization dynamics. It should be noted that strong field IR pulses are often required in attosecond experiments to obtain statistically useful signal. As an estimate, each HHG based XUV pulse only prepares countably few interesting excited atoms/molecules in the focal volume. To ensure that all of this excited sample is indeed probed, the probe needs to have a high flux. Typical probe peak intensities in the range of $10^{12} \mathrm{Wcm}^{-2}$ are often used. Moreover, moderately strong fields are also used in molecular attoscience experiments for alignment, orientation and recollision purposes. Thus, it is pertinent to question how these fields affect the dynamics under investigation. Our work quantitatively measures the dynamic changes in atomic structure and shows that the ionization paths change with intensity and each path has a specific phase associated with it. The knowledge of this dynamic structure and associated phase variation is crucial to understand the dynamics and to devise new control strategies in attoscience.

This work was supported by the National Science Foundation grant PHY-0955274.

\section{References}

[1] F. Krausz, M. Ivanov, Rev. Mod. Phys. 81 (2009) 163-234.

[2] P. B. Corkum, Phys. Rev. Lett. 71 (1993) 1994-1997.

[3] S. Haessler, B. Fabre, J. Higuet, J. Caillat, T. Ruchon, P. Breger, B. Carre, E. Constant, A. Maquet, E. Mevel, P. Salieres, R. Taieb, Y. Mairesse, Physical Review A 80 (2009) -.

[4] A. S. Sandhu, E. Gagnon, R. Santra, V. Sharma, W. Li, P. Ho, P. Ranitovic, C. L. Cocke, M. M. Murnane, H. C. Kapteyn, Science 322 (2008) 10811085.

[5] H. Wang, M. Chini, S. Y. Chen, C. H. Zhang, F. He, Y. Cheng, Y. Wu, U. Thumm, Z. H. Chang, Physical Review Letters 105 (2010) 143002.

[6] P. Eckle, A. N. Pfeiffer, C. Cirelli, A. Staudte, R. Dorner, H. G. Muller, M. Buttiker, U. Keller, Science 322 (2008) 1525-1529.

[7] A. T. J. B. Eppink, D. H. Parker, Review of Scientific Instruments 68 (1997) 3477-3484.

[8] N. Shivaram, A. Roberts, L. Xu, A. Sandhu, Optics Letters 35 (2010) 3312-3314.

[9] P. M. Paul, E. S. Toma, P. Breger, G. Mullot, F. Auge, P. Balcou, H. G. Muller, P. Agostini, Science 292 (2001) 1689-1692.

[10] H. G. Muller, Applied Physics B-Lasers and Optics 74 (2002) S17-S21.

[11] M. Swoboda, T. Fordell, K. Klunder, J. M. Dahlstrom, M. Miranda, C. Buth, K. J. Schafer, J. Mauritsson, A. L'Huillier, M. Gisselbrecht, Physical Review Letters 104 (2010) 103003.

[12] P. Ranitovic, X. M. Tong, C. W. Hogle, X. Zhou, Y. Liu, N. Toshima, M. M. Murnane, H. C. Kapteyn, Physical Review Letters 106 (2011) 193008.

[13] M. Holler, F. Schapper, L. Gallmann, U. Keller, Physical Review Letters 106 (2011) 123601.

[14] L. H. Haber, B. Doughty, S. R. Leone, Physical Review A 79 (2009) 031401.

[15] P. Johnsson, J. Mauritsson, T. Remetter, A. L'Huillier, K. J. Schafer, Phys. Rev. Lett. 99 (2007) 233001. 
[16] P. Ranitovic, et al., New J. Phys. 12 (2010) 013008.

[17] P. Riviere, O. Uhden, U. Saalmann, J. M. Rost, New Journal of Physics 11 (2009) 053011.

[18] S. I. Chu, D. A. Telnov, Phys. Rep. 390 (2004) 1-131.

[19] X. M. Tong, P. Ranitovic, C. L. Cocke, N. Toshima, Physical Review A $81(2010) 021404$.

[20] X. M. Tong, N. Toshima, Phys. Rev. A 81 (2010) 043429.

[21] X. M. Tong, N. Toshima, Physical Review A 81 (2010) 063403.

[22] L. C. Dinu, H. G. Muller, S. Kazamias, G. Mullot, F. Auge, P. Balcou, P. M. Paul, M. Kovacev, P. Breger, P. Agostini, Phys.l Rev. Lett. 91 (2003) 063901.

[23] S. A. Aseyev, Y. Ni, L. J. Frasinski, H. G. Muller, M. J. J. Vrakking, Phys. Rev. Lett. 91 (2003) 223902. 